\title{
Thermodynamic properties of the fluid, fcc, and bcc phases of monodisperse charge-stabilized colloidal suspensions within the Yukawa model
}

\author{
C. F. Tejero \\ Facultad de Ciencias Físicas, Universidad Complutense de Madrid, E-28040 Madrid, Spain \\ J. F. Lutsko \\ Expert Systems Applications Development Group, Departement CIT, Katholieke Universiteit Leuven, \\ B-3001, Heverlee, Belgium \\ J. L. Colot and M. Baus \\ Faculté des Sciences, Case Postale 231, Université Libre de Bruxelles, B-1050 Brussels, Belgium
}

(Received 11 February 1992)

\begin{abstract}
The thermodynamic properties of the Yukawa model for colloidal suspensions are determined theoretically from the Rogers-Young integral equation for the fluid phase and from a recently introduced van der Waals-like theory for the solid phases. Very good agreement with the Monte Carlo simulations of Meijer and Frenkel [J. Chem. Phys. 94, 2269 (1991)] is found for both the fluid and the (fcc-bcc) solid phases. The location of the two-phase coexistences, however, is shown to involve such small free-energy and density changes that no definite statements about the phase diagram are possible within the present accuracy.

PACS number(s): 61.20. $-\mathrm{p}, 64.70 .-\mathrm{p}, 64.30 .+\mathrm{t}$
\end{abstract}

\section{INTRODUCTION}

The experimental study of charge-stabilized colloidal dispersions has made considerable progress in recent years [1]. Monodisperse spherical colloidal particles have been shown to form colloidal crystals. Both bodycentered cubic (bcc) and face-centered cubic (fcc) crystals have been found. The full phase diagram consists of two melting lines (liquid-bcc and liquid-fcc) and a structural (bcc-fcc) transition. The experimental liquid-bcc-fcc triple point has, however, not yet been determined accurately.

Various computer simulation studies have also been undertaken [2]. They have shown that the experimental behavior can be reproduced, at least qualitatively, by assuming that the colloidal particles interact via a spherically symmetric repulsive pair potential of the Yukawa type. Although this represents an oversimplification of the original very complex interactions between the colloidal "particles," most of the experimental trends can nevertheless be reproduced by such simulations. This dramatic simplification can be understood on the basis of the Derjaguin-Landau-Verwey-Overbeek (DLVO) interaction between the spherical electrostatic double layers surrounding the colloidal spheres. When the attractive contribution of the DLVO potential is neglected and the point-particle limit of its repulsive contribution is taken, a simple Yukawa pair potential is obtained. It is this limiting situation that most of the computer simulations have considered. It is supposed to be a good approximation for sufficiently dilute systems. We will refer to it as the Yukawa model for the colloidal dispersions.

In the present investigation we will likewise consider an equilibrium system of point particles interacting via the following purely repulsive Yukawa pair potential $V(r)$ :

$$
V(r)=\varepsilon \frac{\sigma}{r} \exp \left[-\frac{r}{\sigma}\right),
$$

where $r$ is the interparticle distance, while $\varepsilon$ measures the strength and $\sigma$ the range of the interactions. In the real colloidal system both $\varepsilon$ and $\sigma$ are in fact densitydependent quantities, which in this simple model and in the simulations are taken as constants. Once the phase diagram of this model system is quantitatively understood, a corresponding study of the more realistic situation can be undertaken. We now introduce $\beta \varepsilon=1 / t$, with $t$ the reduced temperature $T$ in energy units and $\beta=1 / k_{B} T$ as usual. Similarly, the reduced average density $\rho$ will be written as $\rho \sigma^{3}=1 / \lambda^{3}$, with $\lambda=a / \sigma$ and $a$ the average nearest-neighbor distance $\rho=1 / a^{3}$. The dimensionless potential $\beta V(r)$ can then be written as

$$
\beta V(r)=\frac{1}{t \lambda x} \exp (-\lambda x)
$$

where $x=r / a$ is the reduced distance. The densitytemperature $(\rho-T)$ phase diagram of the resulting Yukawa model will then be represented in the $\lambda-t$ plane.

A number of limited theoretical studies of such a Yukawa system within the density-functional theory of freezing and with various levels of sophistication have already been performed [3]. Here we will consider a much more extensive theoretical study of each of the three phases involved within the most precise theoretical schemes presently available and compare the results to 
the very precise free-energy determinations of Meijer and Frenkel [4]. In Sec. II we will consider the results obtained for the liquid phase from the Rogers-Young integral equation [5]. In Sec. III we use a recently introduced hard-sphere perturbation theory [6] to study the bcc and fcc solid phases while the resulting phase boundaries are discussed in Sec. IV. Our conclusions are then gathered in Sec. V.

\section{FLUID PHASE}

In order to obtain the thermodynamic properties of the Yukawa fluid (or liquid, since only repulsive forces are taken into account), we will use as a starting point the Rogers-Young (RY) integral equation [5]. It has already been shown that this approach yields very accurate results for simple fluids with purely repulsive interactions (the extension to attractive potentials is discussed in Ref. [7]). The RY approximation consists of an interpolation scheme between two well-known approximations of the theory of simple fluids: the Percus-Yevick (PY) and the hypernetted-chain (HNC) approximations. In terms of the pair correlation $g(r)=1+h(r)$, the total correlation $h(r)$ and the direct correlation $c(r)$ functions, the RY equations consist of the Ornstein-Zernike equation

$$
h(r)=c(r)+\rho \int d \mathbf{r}^{\prime} h\left(r^{\prime}\right) c\left(\left|\mathbf{r}-\mathbf{r}^{\prime}\right|\right),
$$

where $r=|\mathbf{r}|$, and the RY closure relation

$g(r)=\left(1+\frac{\exp [\gamma(r) f(r)]-1}{f(r)}\right) \exp [-\beta V(r)]$,

where $\gamma(r)=h(r)-c(r), \beta V(r)$ is the reduced interaction potential given here by Eq. (1.1), and $f(r)$ is an exponential interpolation function such that for $f(r)=0$ or $1 \mathrm{Eq}$. (2.2) restores, respectively, the PY or the HNC closure relation. For $f(r)$ we take, in the reduced variables of Sec. I $(x=r / a)$ :

$$
f(r)=1-\exp (-\alpha x),
$$

where $\alpha$ is a dimensionless parameter $(0 \leq \alpha \leq \infty)$ still to be determined. We first numerically solve Eqs. (2.1) - (2.3) for a given $\alpha$ value with the aid of Gillan's algorithm [8]. In terms of the reduced variables of Sec. I, the solution $g(x)$ or $c(x)$ will be a function of the reduced temperature $t$, the reduced density or the reduced average interparticle distance $\lambda$, and the dimensionless interpolation parameter $\alpha$.

To test the quality of the numerical solution so obtained we compare the following two expressions for the isothermal compressibility modulus $B=\beta(\partial P / \partial \rho)_{T}, P$ being the pressure:

$$
B_{c}=1-4 \pi \int_{0}^{\infty} d x x^{2} c(x),
$$

which is the value of $B$ obtained from $c(x)$, and

$$
B_{h}=\left(1+4 \pi \int_{0}^{\infty} d x x^{2} h(x)\right]^{-1},
$$

i.e., the value of $B$ obtained from $h(x)$. The numerical solutions are accepted whenever $\left|B_{c}-B_{h}\right| / B_{c}<0.005$ is realized. We found that for the present system (1.2), the convergence of Gillan's algorithm is fairly slow, especially for the high densities close to freezing. In some cases, up to 200 iterations were required. We also found that changing the interpolation function [5] does not appreciably improve the situation and we will therefore always stick to the exponential form of (2.3). In the final step one still has to fix the interpolation parameter $\alpha$ in such a manner as to obtain thermodynamic consistency. Indeed, since the RY closure (2.2) is not exact, different routes to the pressure $P$ will give different results. One can compute then the compressibility factor $Z=\beta P / \rho$ from $c(x)$ using (2.4) as

$$
Z_{c}=\frac{1}{\rho} \int_{0}^{\rho} d \rho^{\prime} B_{c}\left(\rho^{\prime}\right),
$$

or from the virial equation, which for (1.2) reads

$Z_{v}=1+\frac{2 \pi}{3 t \lambda} \int_{0}^{\infty} d x x g(x)(1+\lambda x) \exp (-\lambda x)$,

to which there will correspond now a virial compressibility modulus $B_{v}$ :

$$
B_{v}=Z_{v}+\rho \frac{\partial Z_{v}}{\partial \rho} .
$$

Once a consistent pressure has been found, the Helmholtz free energy per particle $f$ can be obtained from

$$
\beta f=\ln \left(\Lambda^{3} \rho\right)-1+\int_{0}^{\rho} \frac{d \rho^{\prime}}{\rho^{\prime}}\left[Z\left(\rho^{\prime}\right)-1\right],
$$

where $\Lambda$ is the thermal de Broglie wavelength, and the chemical potential $\mu$ follows then from

$$
\beta \mu=\beta f+Z \text {. }
$$

Everything amounts thus to finding a thermodynamically consistent pressure and to compute $f$ and $\mu$ from it. Ideally one would like to have $Z_{c}=Z_{v}$ and $B_{c}=B_{v}$, since we have already realized $B_{c}=B_{h}$ (implying $Z_{c}=Z_{h}$ ) in the first step. In the original RY method [5], one determines $\alpha$ in such a manner that $\left|B_{v}-B_{c}\right| / B_{c}<0.005$ (remember that $\left|B_{h}-B_{c}\right| B_{c}<0.005$ is realized here for every $\alpha$ value), i.e., the thermodynamic consistency is realized on the compressibility modulus $B$. The utility of the RY method is largely due to the observation that for simple repulsive potentials, such as the inverse-power potentials considered in [5], the interpolation parameter $\alpha$, which realizes this $B$ consistency for a given potential, is almost density independent. We found, however, that for the Yukawa potential (1.2) this is not the case for the range of densities considered here. The resulting variation of $\alpha$ with the density makes the procedure much more difficult to implement, so that a substantial numerical effort is required for each temperature and density in order to achieve $\boldsymbol{B}$ consistency. Moreover, we found that there is an important residual $Z$ inconsistency. Indeed, computing $\alpha=\alpha(\lambda, t)$ from the original RY prescription $\left|B_{c}-B_{v}\right| / B_{c}<0.005$, with a full account of the density dependence of $\alpha(\lambda, t)$ while computing $B_{v}$, we find that the resulting pressures can still differ considerably in the 
high-density region close to freezing. Typically we find $\left|Z_{c}-Z_{v}\right| Z_{v} \simeq 0.1$ in this region. This is presumably due to a systematic accumulation of small errors while computing $Z_{c}$ which involves an integral over the density dependence of $\alpha$. Hence, even a small $B$ inconsistency can end up in a much larger $Z$ inconsistency leading via (2.9) to even larger $\beta f$ inconsistencies. In order to avoid this problem we have used the following alternative implementation of the RY method. First we compute, for a given temperature $(t)$ and density $(\lambda), B_{c}=B_{c}(t, \lambda ; \alpha)$ from (2.4) and $Z_{v}=Z_{v}(t, \lambda ; \alpha)$ from (2.7) as functions of the free parameter $\alpha$. Next we fit these results to a polynomial in $\alpha$. Then we consider $Z_{v}=Z_{v}(t, \lambda ; \alpha)$ for fixed $t$ and $\alpha$ and fit the result to a rational expression in the density $\left(=1 / \lambda^{3}\right)$. From this rational expression for $Z_{v}$ we compute analytically $B_{v}$ and then equate the result to the polynomial fit of $B_{c}$. In this way we realize simultaneously both the $B$ consistency and the $Z$ consistency, but now only for the chosen fitting functions. We then check that the results do not appreciably depend on the chosen fitting functions. In the present case we could realize this program by taking a polynomial of order eight for the $\alpha$ fits and a rational expression consisting of the ratio of two second-order polynomials for the density fits. Thus the fits were realized numerically by using an eight-point grid covering the $\alpha$ range and a four-point grid covering the density range. The total numerical effort involved in this procedure is much smaller than for the original RY implementation, but it should be realized that the full consistency is now achieved only on a discrete grid of points and for the chosen fitting functions. We will henceforth refer to the results obtained in this way as resulting from the discretized RY method (abbreviated below as DRY).

We now compare the results obtained from the above RY and DRY methods to the Monte Carlo (MC) simulations performed by Meijer and Frenkel [4] for the Yukawa system (1.1) and (1.2). In Fig. 1 we compare the re-

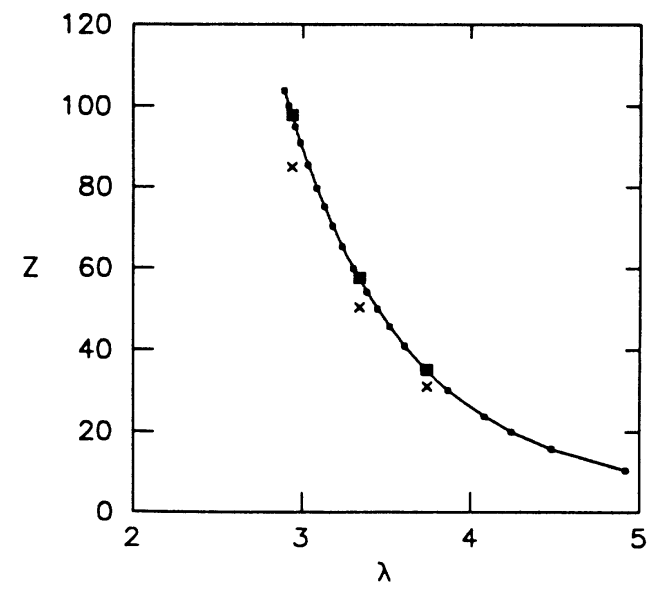

FIG. 1. The compressibility factor $Z=\beta P / \rho$ of the fluid phase vs $\lambda$ for $t=1.4 \times 10^{-3}$ as obtained from the DRY method (solid line). The MC results [4] are denoted by full dots while the results from the original RY method are displayed by squares $\left(Z_{v}\right)$ and crosses $\left(Z_{c}\right)$.
TABLE I. The compressibility factor $Z=\beta P / \rho$ for the fluid phase as a function of $\lambda$ for $t=1.4 \times 10^{-3}$ as obtained from the DRY method $\left(Z_{d}\right)$ compared to the simulations $\left(Z_{\mathrm{MC}}\right)$ of Meijer and Frenkel [4].

\begin{tabular}{crr}
\hline \hline$\lambda$ & $Z_{\mathrm{MC}}$ & \multicolumn{1}{c}{$Z_{d}$} \\
\hline 2.901 & 102.492 & 102.751 \\
2.999 & 89.606 & 89.846 \\
3.100 & 78.148 & 78.387 \\
3.198 & 68.640 & 68.865 \\
3.303 & 59.889 & 60.091 \\
3.412 & 52.133 & 52.307 \\
3.518 & 45.711 & 45.862 \\
3.607 & 41.041 & 41.176 \\
3.718 & 35.954 & 36.072 \\
3.785 & 33.204 & 33.314 \\
3.865 & 30.294 & 30.394 \\
4.081 & 23.780 & 23.855 \\
4.241 & 20.016 & 20.069 \\
4.478 & 15.705 & 15.722 \\
4.917 & 10.400 & 10.343 \\
\hline \hline
\end{tabular}

sults for the compressibility factor $Z$ as obtained from the RY and DRY methods to the simulation results at the highest temperature. It is seen that as stated above, the residual thermodynamic inconsistency between $Z_{v}$ and $Z_{c}$ as obtained from the RY method is considerable, and that as expected, $Z_{v}$ gives the best agreement with the simulations. It can also be observed that the $Z_{v}$ values of the RY method are close to the results, say $Z_{d}$, of the DRY method $\left(\left|Z_{v}-Z_{d}\right| Z_{d}<0.007\right)$, both of which are very close to the simulation results $Z_{\mathrm{MC}}$ with $\left|Z_{\text {MC }}-Z_{d}\right| Z_{d}<0.003$. In Table $I$ we compare the $Z$ values for the highest temperature considered in the simulations, while Fig. 2 displays the free energies for the four temperatures for which simulations have been performed. The agreement between the theory and the simulations is very good in all four cases.

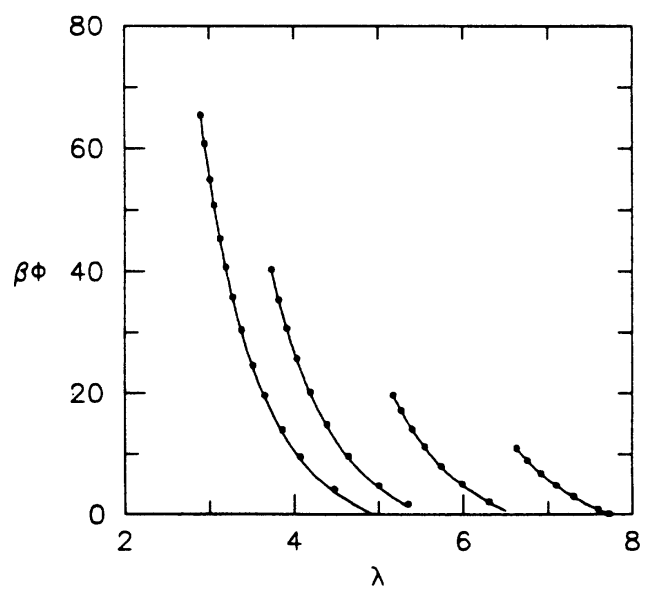

FIG. 2. The free energy per particle $\beta \phi$ of the fluid phase as obtained from the DRY method (solid lines) vs $\lambda$ for (from right to left) $t=4.3 \times 10^{-5}, 1.7 \times 10^{-4}, 6.3 \times 10^{-4}$, and $1.4 \times 10^{-3}$. The full dots are the MC simulations of Meijer and Frenkel [4]. 


\section{SOLID PHASES}

For the theoretical determination of the thermodynamic properties of the solid phase of the Yukawa system, we will use a recently proposed [6] hard-sphere (HS) perturbation theory. Elsewhere [6], this theory was shown to yield accurate results for the solid phases of the inversepower potentials. This theory is based on a straightforward extension to the solid phase of the ideas behind the classical van der Waals theory of the fluid phase. Its basic result is an expression for the Helmholtz free energy per particle $f$ of the solid from which the compressibility factor $Z=\beta P / \rho$ can then be obtained by differentiation according to

$$
Z=\beta \rho \frac{\partial f}{\partial \rho},
$$

while the chemical potential $\mu$ then follows from (2.10). The approximate expression for $f$ has a typical van der Waals structure [6]:

$$
f=f_{\mathrm{HS}}(d)+\frac{1}{2} \int_{r>d} d \mathbf{r} \rho_{0}(r) W(r),
$$

where the first term $f_{\mathrm{HS}}(d)$ represents an "equivalent" hard-sphere contribution that originates from splitting the original potential $V(r)=V_{0}(r)+W(r)$ into a harsh short-ranged reference potential $V_{0}(r)$ and a weak longranged perturbation $W(r)$. One obvious difference with the original van der Waals theory is that here $f_{\mathrm{HS}}(d)$ refers to the HS solid instead of the HS fluid. The second term of (3.2) reduces to a static lattice sum

$$
\frac{1}{2} \int_{r>d} d \mathbf{r} \rho_{0}(r) W(r)=\frac{1}{2} \sum_{r_{j}(>d)} W\left(r_{j}\right),
$$

where $\rho_{0}(r)=\Sigma_{j} \delta\left(\mathbf{r}-\mathbf{r}_{j}\right)$ describes the distribution of the lattice sites $\mathbf{r}_{j}$ around the central site at the origin (i.e., $\left.\mathbf{r}_{0}=0\right)$ and $r_{j}=\left|\mathbf{r}_{j}\right|$. When this distribution is smeared out, $\rho_{0}(\mathbf{r}) \rightarrow \rho$; to give a uniform fluid, Eq. (3.3) is seen to reduce to the usual fluid-phase van der Waals term $\frac{1}{2} \rho \int_{r>d} d \mathbf{r} W(r)$, except that here $W(r)$ will describe a (long-ranged) repulsive perturbation instead of the usual attractive perturbation used in the original van der Waals theory. Notice also that in the derivation [6] of (3.2) we have taken advantage of the fact that in the solid phase the structural properties (e.g., the layering of the particles) are dominated by the one-body density, while the main purpose of the residual pair correlations is to prevent the particles from penetrating the hard core of diameter $d$ surrounding each of them [hence the restriction $r>d$ in (3.2)]. In the fluid phase the corresponding layering of the particles is described instead by the paircorrelation function (for $r>d$ ) and the latter cannot be neglected so that (3.2) will only be applicable to the solid phase. A hard-sphere perturbation theory for the fluid phase can nevertheless also be constructed but turns out to be less accurate than the results obtained in the previous section from the Rogers-Young integral equation.

The final implementation of (3.2) still requires a prescription for $f_{\mathrm{HS}}(d), d$, and $V_{0}(r)$. For $f_{\mathrm{HS}}(d)$ we will use the numerical results obtained elsewhere [9] from the HS density-functional theory of freezing within the "gen- eralized effective liquid approximation" (GELA). For convenience, the HS-GELA results are given in Table II as a function of the packing fraction $\eta=(\pi / 6) d^{3} \rho$ for both the fcc and the bcc HS phases. The equivalent HS diameter $d$ can now be related to the reference potential $V_{0}(r)$ by the Weeks-Chandler-Andersen prescription [10]. When, as proposed by Verlet-Weis [11], this value is expanded around the Barker-Henderson $(\mathrm{BH})$ approximation [12] $d_{\mathrm{BH}}$, we obtain upon retaining only the first correction $\delta$ the following relation between $d$ and $V_{0}(r)$ :

$$
\begin{aligned}
& d=d_{\mathrm{BH}}\left(1+\frac{\delta}{2}\right), \\
& d_{\mathrm{BH}}=\int_{0}^{\infty} d r\left\{1-\exp \left[-\beta V_{0}(r)\right]\right\}, \\
& \delta=\int_{0}^{\infty} d r\left(1-\frac{r}{d_{\mathrm{BH}}}\right)^{2} \frac{d}{d r}\left\{\exp \left[-\beta V_{0}(r)\right]\right\} .
\end{aligned}
$$

Notice the misprints in the expression corresponding to (3.4) in Ref. [5]. For consistency, the higher-order corrections in (3.4a) have been neglected because they involve the pair correlations of the HS solid, which have already been neglected in the derivation of (3.2). Moreover, the first-order correction $\delta$ of $(3.4 a)$ is itself only a small correction to $d_{\mathrm{BH}}$ so that essentially the same results can be obtained by using $d_{\mathrm{BH}}$ as HS diameter. Finally, for the reference potential $V_{0}(r)$ we have adopted the Kang-Lee-Ree-Ree prescription [13], so that $V_{0}(r)$ is of finite range $r_{0}$ with zero slope at $r=r_{0}$. This range parameter $r_{0}$ will be set equal to the nearest-neighbor distance in a compact lattice structure, which is thus used here as a reference structure whatever the final lattice to be studied. The resulting expression of $V_{0}(r)$ correspond-

TABLE II. The Helmholtz free energy per particle $f_{\mathrm{HS}}$ of the (fcc or bcc) hard-sphere (HS) solid is given by $\beta f_{\mathrm{HS}}=\beta \phi_{\mathrm{HS}}-3 \ln (d / \Lambda)-1$, where $\Lambda$ is the thermal de Broglie wavelength and $d$ the HS diameter. The quantity $\beta \phi_{\mathrm{HS}}$ depends only on the packing fraction $\eta=(\pi / 6) \rho d^{3}$. These results have been obtained from the GELA [9] by using the (fluid-phase) HS direct correlation function given in [15].

\begin{tabular}{ccc}
\hline \hline$\eta$ & $\beta \phi_{\mathrm{HS}}(\mathrm{fcc})$ & $\beta \phi_{\mathrm{HS}}(\mathrm{bcc})$ \\
\hline 0.48 & 3.6889 & 3.4982 \\
0.49 & 3.8878 & 3.7286 \\
0.50 & 4.0816 & 3.9696 \\
0.51 & 4.2760 & 4.2225 \\
0.52 & 4.4729 & 4.4585 \\
0.53 & 4.6730 & 4.7015 \\
0.54 & 4.8775 & 4.9547 \\
0.55 & 5.0875 & 5.2210 \\
0.56 & 5.3040 & 5.5033 \\
0.57 & 5.5280 & 5.8049 \\
0.58 & 5.7609 & 6.1300 \\
0.59 & 6.0041 & 6.4831 \\
0.60 & 6.2592 & 6.8703 \\
0.61 & 6.5284 & 7.2980 \\
0.62 & 6.8142 & 7.7742 \\
0.63 & 7.1196 & 8.3078 \\
\hline \hline
\end{tabular}




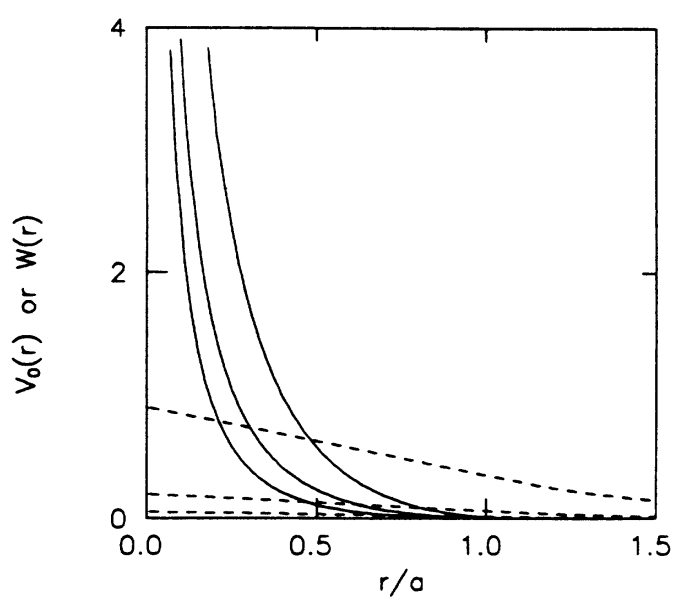

FIG. 3. Separation of the Yukawa potential as described by Kang-Lee-Ree-Ree [13] vs $r / a$ for $\lambda=1,2,3$. The reference potential $V_{0}(r)$ (solid lines) is from right to left and the perturbation $W(r)$ (dashed lines) is from top to bottom, respectively.

ing to the Yukawa potential $V(r)$ of (1.1) and (1.2) reads then

$$
\begin{aligned}
\beta V_{0}(r)=\Theta\left(x_{0}-x\right) \frac{1}{t \lambda}[ & \frac{\exp (-\lambda x)}{x} \\
+ & {\left[\left(x-x_{0}\right)\left(1+\lambda x_{0}\right)-x_{0}\right] } \\
& \left.\times \frac{\exp \left(-\lambda x_{0}\right)}{x_{0}^{2}}\right],
\end{aligned}
$$

where, as above, $x=r / a$ and $x_{0}=r_{0} / a \equiv 2^{1 / 6}$ and $\Theta(x)=1$ for $x>0$ and zero otherwise. Using (3.5) in (3.4) one obtains $d=d(t, \lambda)$, which is then used to evaluate (3.2) with the aid of Table II for $f_{\mathrm{HS}}(d)$, while the lattice sums of (3.3) can be performed using the Ewald summation method of Medeiros e Silva and Mokross [14]

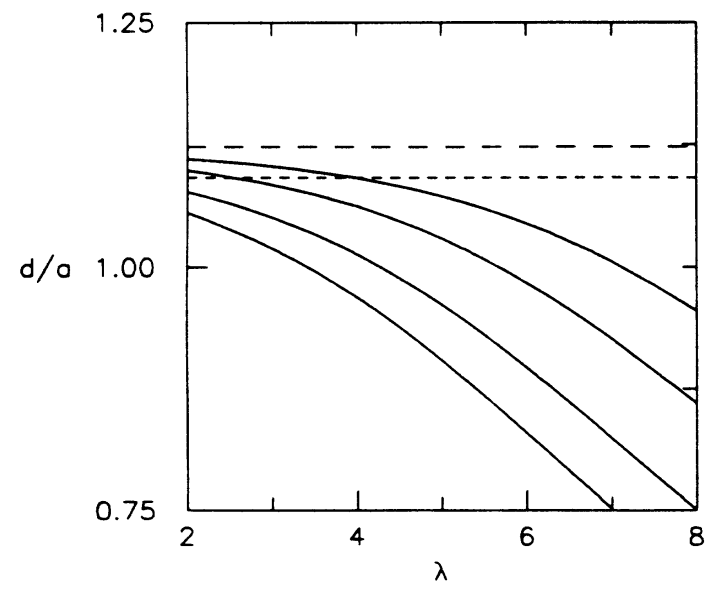

FIG. 4. The equivalent HS diameter $d / a$ vs $\lambda$ (solid lines) for (from top to bottom) $t=4.3 \times 10^{-5}, 1.7 \times 10^{-4}, 6.3 \times 10^{-4}$, and $1.4 \times 10^{-3}$. The short-dashed line (medium-dashed line) is the distance of the first shell from the central particle $\left(r_{1}\right)$ measured in terms of $a\left(x_{1}=r_{1} / a\right)$ for the bcc (fcc) lattice.

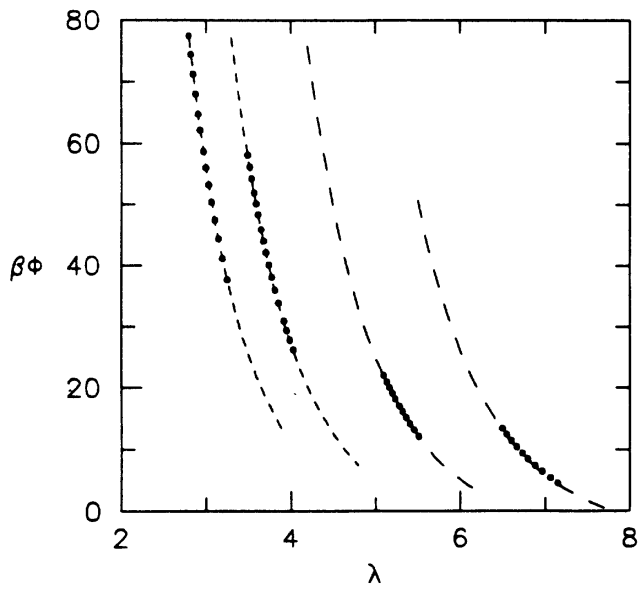

FIG. 5. The free energy $\beta \phi$ per particle of the solid phase vs $\lambda$ for the fcc lattice (medium-dashed lines) for (from right to left) $t=4.3 \times 10^{-5}$ and $1.7 \times 10^{-4}$ and for the bcc lattice (dashed lines) for (from right to left) $6.3 \times 10^{-4}$ and $1.4 \times 10^{-3}$. The simulation data denoted by full dots are taken from Meijer and Frenkel [4].

(beware of the misprints in Ref. [14]), leading finally to our theoretical predictions for the free energy per particle $f(t, \lambda)$ of the fcc and bcc Yukawa solid phases. The separation of the Yukawa potential resulting from (3.5), $V(r)=V_{0}(r)+W(r)$, is illustrated in Fig. 3, while the behavior of the equivalent HS diameter obtained from (3.4), $d=d(t, \lambda)$, is displayed in Fig. 4 .

We now compare the theoretical results obtained from (3.1) and (3.2) to the MC simulations of Meijer and Frenkel [4]. In Figs. 5 and 6 we compare, respectively, the free energies $(f)$ and the pressures $(Z)$ along the four isotherms considered in the simulations. In view of the simple van der Waals approximation embodied in (3.2), the agreement is surprisingly good both for the fcc and

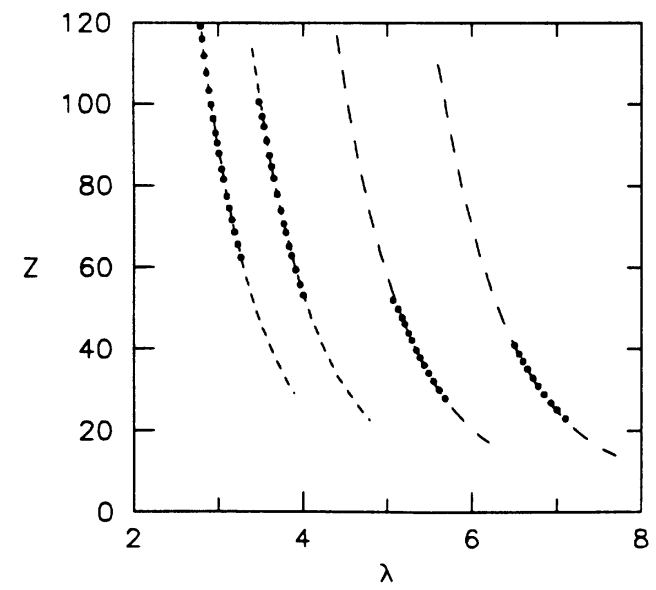

FIG. 6. The compressibility factor $Z=\beta P / \rho$ of the solid phase vs $\lambda$ for the fcc lattice (medium-dashed lines) for (from right to left) $t=4.3 \times 10^{-5}$ and $1.7 \times 10^{-4}$ and for the bcc lattice (dashed lines) for (from right to left) $6.3 \times 10^{-4}$ and $1.4 \times 10^{-3}$. The simulation data of Meijer and Frenkel [4] are denoted by full dots. 
TABLE III: The compressibility factor $Z=\beta P / \rho$ for the fcc solid phase as a function of $\lambda$ for $t=4.3 \times 10^{-5}$ as obtained from Eqs. (3.1)-(3.3) $(Z)$ compared to the simulations $\left(Z_{M C}\right)$ of Meijer and Frenkel [4].

\begin{tabular}{ccc}
\hline$\lambda$ & $Z_{\mathrm{MC}}$ & $Z$ \\
\hline 6.503 & 41.058 & 40.865 \\
6.555 & 38.927 & 38.715 \\
6.602 & 37.115 & 36.871 \\
6.654 & 35.259 & 35.003 \\
6.721 & 32.969 & 32.695 \\
6.783 & 31.005 & 30.721 \\
6.853 & 28.991 & 28.685 \\
6.932 & 26.919 & 26.576 \\
7.003 & 25.211 & 24.834 \\
7.103 & 22.996 & 22.612 \\
\hline \hline
\end{tabular}

the bcc phases. A numerical example is given in Table III. The relative difference between the theoretical and the simulation free energies is always smaller than $3 \%$ with the theoretical results always below the simulation results.

\section{TWO-PHASE COEXISTENCES}

We now compare the three phases (fluid, fcc, and bcc) two by two. First there is the fluid-fcc coexistence. Only two points have been computed in the simulations. Both theory and simulations predict a very weak density change of freezing. For $t=1.7 \times 10^{-4}$ we find the coexistence at $\lambda_{\text {fcc }}=5.48$ and $\lambda_{\text {fluid }}=5.51$, corresponding to a pressure of $\beta \sigma^{3} P=0.21$, whereas the corresponding simulation results [4] are $\lambda_{\text {fcc }}^{\mathrm{MC}}=5.33, \lambda_{\text {fluid }}^{\mathrm{MC}}=5.39$, and $\beta \sigma^{3} P^{\mathrm{MC}}=0.27$. Hence, whereas the density changes are comparable, the transition has been shifted to lower densities as a result of the existence of a small free-energy difference between the theoretical and simulation results for the fcc phase. A similar but worse behavior is found for the fluid-bcc coexistence, as can be seen from Fig. 7.

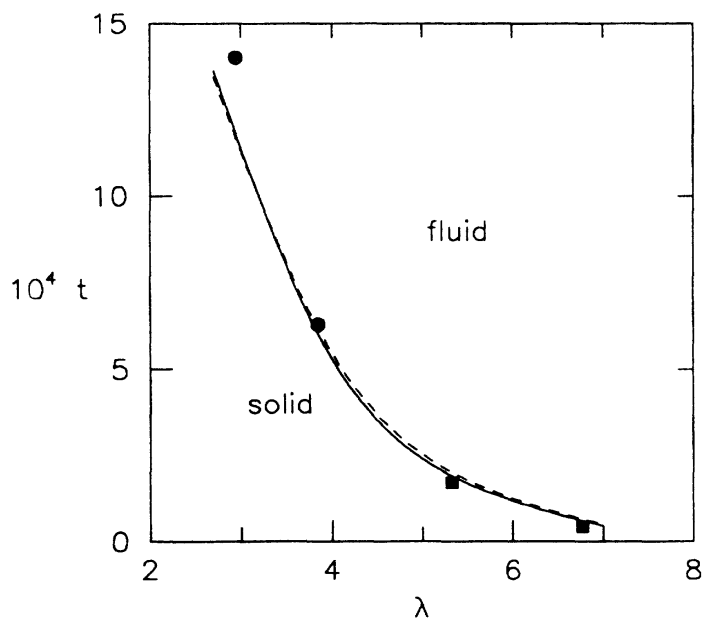

FIG. 7. The fluid-bcc (dashed line) and fluid-fcc (solid line) melting lines in the $\lambda-t$ plane as obtained from the present theory. The MC results of Meijer and Frenkel [4] are denoted by circles (bcc) and squares ( $\mathrm{fcc}$ ) correspond to the average interparticle distance for the solid at coexistence. There is a clear discrepancy at the highest temperature.

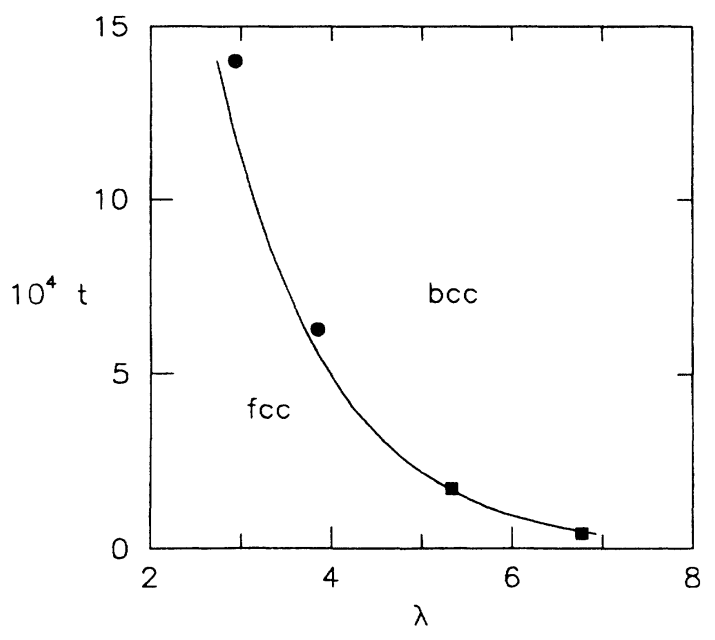

FIG. 8. Structural fcc-bcc transition as obtained from the present theory. The MC data of Fig. 7 are also included here in order to show that the fcc structure assumed in the simulations is only metastable at $t=1.7 \times 10^{-4}$ when viewed from the present theory.

Finally we consider the fcc-bcc coexistence, for which no simulations have been performed by Meijer and Frenkel, although some more approximate estimates can be obtained from the MD simulations of Robbins, Kremer, and Grest [2]. Again we find a very small density change (see Table IV). The position of the structural fcc-bcc transition in the $(t, \lambda)$ plane is quite sensitive to small changes. For instance, we find (see Fig. 8) that along the isotherm $t=1.7 \times 10^{-4}$, where the simulations have assumed the fcc phase to be the equilibrium phase, the latter is still weakly metastable relative to the bcc phase, at least within the present theory. In fact, the free-energy differences involved are so small that they can easily compete with the finite-size effects of the simulations and it becomes very hazardous to make any definite statement as to where the phase boundaries and in particular the (fluid-fcc-bcc) triple point are really located. This will hence not only have to wait for a more precise theory but presumably also for a more precise study of the finite-size effects on the simulation results.

\section{CONCLUSIONS}

We have determined the thermodynamic properties of charge-stabilized colloidal dispersions within the Yukawa model. For the fluid phase the calculations have been based on the Rogers-Young integral equation [5], which

TABLE IV: Coexistence data for the structural (fcc-bcc) transition for the four temperatures considered in the MC simulations of Meijer and Frenkel [4].

\begin{tabular}{cccc}
\hline \hline $10^{4} t$ & $\lambda(\mathrm{fcc})$ & $\lambda(\mathrm{bcc})$ & $\beta P \sigma^{3}$ \\
\hline 14 & 2.735 & 2.736 & 6.299 \\
6.3 & 3.703 & 3.705 & 1.515 \\
1.7 & 5.299 & 5.306 & 0.280 \\
0.43 & 6.920 & 6.936 & 0.081 \\
\hline \hline
\end{tabular}


is known to yield very good results for the inverse-power repulsive potentials. For the Yukawa potential, the implementation of the Rogers-Young method is more complicated, as discussed above in Sec. II, but the results obtained compare very well with the MC computer simulations performed by Meijer and Frenkel [4]. For the fcc and bcc solid phases, we have used a van der Waals-like hard-sphere perturbation theory [6], which was shown previously to yield very good results for the inversepower repulsive potentials. Its extension to the Yukawa potential is straightforward, and as shown in Sec. III, the results obtained in this way compare very favorably with the simulations of the solid phases performed by Meijer and Frenkel [4]. When the free energies of the different phases are compared in order to construct the two-phase coexistence lines, it is found that at coexistence, the freeenergy differences and density differences involved are extremely small. It is concluded that the precise location of the phase transitions of the Yukawa system will require free energies of a precision that exceeds the already considerable accuracy of the present theoretical calculations. We also suggest that the effect of finite-size effects on the computer simulations should be investigated in detail before a definitive conclusion can be reached as to the location of the phase boundaries and in particular of the fluid-bcc-fcc triple point.

\section{ACKNOWLEDGMENTS}

We thank E. J. Meijer for sending us his unpublished simulation data. C. F. T. wishes to thank the Dirección General de Investigatión Científica y Técnica (Spain) (PB88-0140) for the financial support. M. B. acknowledges financial support from the Fonds National de la Recherche Scientifique.
[1] See S. Hachisu, Y. Kobayashi, and A. Kose, J. Colloid Interface Sci. 42, 342 (1973); Y. Monovoukas and A. Gast, ibid. 128, 53 (1989); T. Okubo, J. Chem. Phys. 95, 3690 (1991), and references therein.

[2] See M. O. Robbins, K. Kremer, and G. G. Grest, J. Chem. Phys. 88, 3286 (1988); D. Thirumalai, J. Phys. Chem. 93, 5637 (1989); E. J. Meijer and D. Frenkel, J. Chem. Phys. 94, 2269 (1991), and references therein.

[3] See B. B. Laird and D. M. Kroll, Phys. Rev. A 42, 4810 (1990); S. Sengupta and A. K. Sook, ibid. 44, 1233 (1991); P. Salgi and R. Rajagopalan, Langmuir (to be published).

[4] E. J. Meijer and D. Frenkel, see Ref. [2]; and (private communication).

[5] F. J. Rogers and D. A. Young, Phys. Rev. A 30, 999 (1984).

[6] J. F. Lutsko and M. Baus, J. Phys. Condens. Matter 3,
6547 (1991).

[7] G. Zerah and J. P. Hansen, J. Chem. Phys. 84, 2336 (1986).

[8] M. J. Gillan, Mol. Phys. 38, 1781 (1979).

[9] J. F. Lutsko and M. Baus, Phys. Rev. A 41, 6647 (1990).

[10] J. D. Weeks, D. Chandler, and H. C. Andersen, J. Chem. Phys. 54, 5237 (1971).

[11] L. Verlet and J. J. Weis, Phys. Rev. A 5, 939 (1972).

[12] J. A. Barker and D. Henderson, J. Chem. Phys. 47, 4714 (1967).

[13] H. S. Kang, S. Lee, T. Ree, and F. H. Ree, J. Chem. Phys. 82, 414 (1985).

[14] J. Medeiros e Silva and B. J. Mokross, Phys. Rev. B 21, 2972 (1980).

[15] M. Baus and J. L. Colot, Phys. Rev. A 36, 3912 (1987). 